destination where newly generated peptide pools are made available for binding to them. Absent from the discussion is the obvious consideration of protease specificity: if lysosomal proteases that generate the class II MHC ligands were to display preferred cleavage sites, then the pools of peptides made available to class II molecules would be biased. However, this has never been a popular notion in view of the redundancy, in terms of substrate specificity, of lysosomal proteases. There are examples to the contrary, however: asparagine-specific endoprotease is indeed remarkably selective in the peptide bonds it cleaves, and the enzyme has been implicated in the generation, in a highly selective fashion, of class II MHC-restricted epitopes (4). Nonetheless, there are very few examples of antigens for which the processing pathway has been characterized comprehensively in terms of intermediates and the proteases that generate them. It may well be that the convergence in peptide binding, observed for DQ8 and I-Ag7, produced by the identical types of antigen-presenting cells, owes at least a component of this repertoire to shared enzymatic machinery upstream. As Suri et al. state (1), expression levels of the source antigen and the possibilities of encounter with the entire processing and peptide loading apparatus are certainly going to affect the final outcome, in terms of the MHC-peptide complexes produced. The best map of a landscape is the landscape itself: seen from this perspective, the comprehensive data set presented here by Suri and coworkers will allow others to navigate the territory of autoimmunity with an accurate map in hand.
Address correspondence to: Hidde L. Ploegh, Department of Pathology, Harvard Medical School, 77 Avenue Louis Pasteur, Boston, Massachusetts 02115, USA. Phone: (617) 432-4776; Fax: (617) 432-4775; E-mail: ploegh@hms.harvard.edu.

1. Suri, A., Walters, J.J., Gross, M.L., and Unanue, E.R. 2005. Natural peptides selected by diabetogenic DQ8 and murine I-Ag7 molecules show common sequence specificity. J. Clin. Invest. 115:2268-2276. doi:10.1172/JCI25350.

2. Kast, W.M., et al. 1991. Protection against lethal Sendai virus infection by in vivo priming of virus-specific cytotoxic $T$ lymphocytes with a free synthetic peptide. Proc. Natl. Acad. Sci. U. S. A. 188:2283-2287.

3. Kent, S.C., et al. 2005. Expanded T cells from pancreatic lymph nodes of type 1 diabetic subjects recognize an insulin epitope. Nature. 435:224-228.

4. Antoniou, A.N., Blackwood, S.L., Mazzeo, D., and Watts, C. 2000. Control of antigen presentation by a single protease cleavage site. Immunity. 12:391-398.

\title{
Of mice and men: the iron age
}

\author{
Sophie Vaulont, Dan-Qing Lou, Lydie Viatte, and Axel Kahn
}

Institut Cochin, INSERM 567, CNRS 8104, Université Paris 5, Paris, France.

\begin{abstract}
Recently, mutations causing juvenile hemochromatosis have been identified in a novel gene, hemojuvelin (HJV), located on chromosome 1. Mouse models of this disease have now been developed by 2 groups, Huang et al. and Niederkofler et al., through targeted disruption of the $\mathrm{Hj} v$ gene (see the related articles beginning on pages 2180 and 2187). These mutant mice will allow further investigation into the role of $\mathrm{HJV}$ in the regulation of iron homeostasis, a role that to date remains elusive.
\end{abstract}

The following scenario is classic with respect to genetic disorders: the disease is clinically recognized, the related gene is identified, and a couple of years later, a mouse model of the genetic disease is established. The genes involved in iron metabolism have not been an exception to this rule (in particular those related to hereditary hemochromatosis, a prevalent heterogeneous iron-overload disorder), and the development of mouse models have greatly facilitated our understanding of the pathogenesis of iron overload. The hemojuvelin gene (HJV, also known as HFE2 and RGMc), which is mutated in a

Nonstandard abbreviations used: HAMP, hepcidin antimicrobial peptide; HJV, hemojuvelin; TFR2, transferrin receptor 2 .

Conflict of interest: The authors have declared that no conflict of interest exists.

Citation for this article: J. Clin. Invest. 115:2079-2082 (2005). doi:10.1172/JCI25642. form of juvenile hemochromatosis linked to chromosome 1, was identified in 2003 (1). Researchers in the field have therefore been waiting for the development of a relevant mouse model of this disease. This feat has now been accomplished by Huang et al. and Niederkofler et al., who in this issue of the JCI describe the phenotype of their mutant mice $(2,3)$. Their findings add further support to the emerging attractive hypothesis that hepcidin, a recently described iron-regulatory peptide, and ferroportin, its target, are common pathogenic denominators in all forms of iron overload.

In mammals, iron homeostasis is complex and depends on regulated dietary iron absorption by mature enterocytes of the duodenum and iron recycling by macrophages, which supply most of the serum iron through recovery of the metal from senescent erythrocytes (4). These 2 fundamental processes are deregulated in hereditary hemochromatosis; over time, this deregulation leads to iron deposition in parenchymal cells and transferrin saturation (5). As the disease progresses, patients develop iron-induced tissue damage, which results in serious illnesses including cirrhosis, hepatomas, diabetes, cardiomyopathy, arthritis, and endocrinopathies. Unless recognized and treated, this disease is fatal.

While hereditary hemochromatosis has been clinically recognized since 1889 , it is only over the 10 past years that the genetic basis for the disease has been elucidated. Hereditary hemochromatosis is now recognized to be genetically heterogeneous and its nomenclature is under debate. Depending on distinct clinical and genetic entities, hereditary hemochromatosis can be divided into 3 classes: classical hemochromatosis, juvenile hemochromatosis, and ferroportin disease.

Classical hemochromatosis is associated with the historical and most prevalent form of hereditary hemochromatosis and is almost always caused by mutations in $H F E$, a gene which encodes a protein of the major histocompatibility complex class I (6). Rarely, another gene can be involved, namely transferrin receptor 2 (TFR2) (7), which encodes a homolog of TFR1 that mediates 


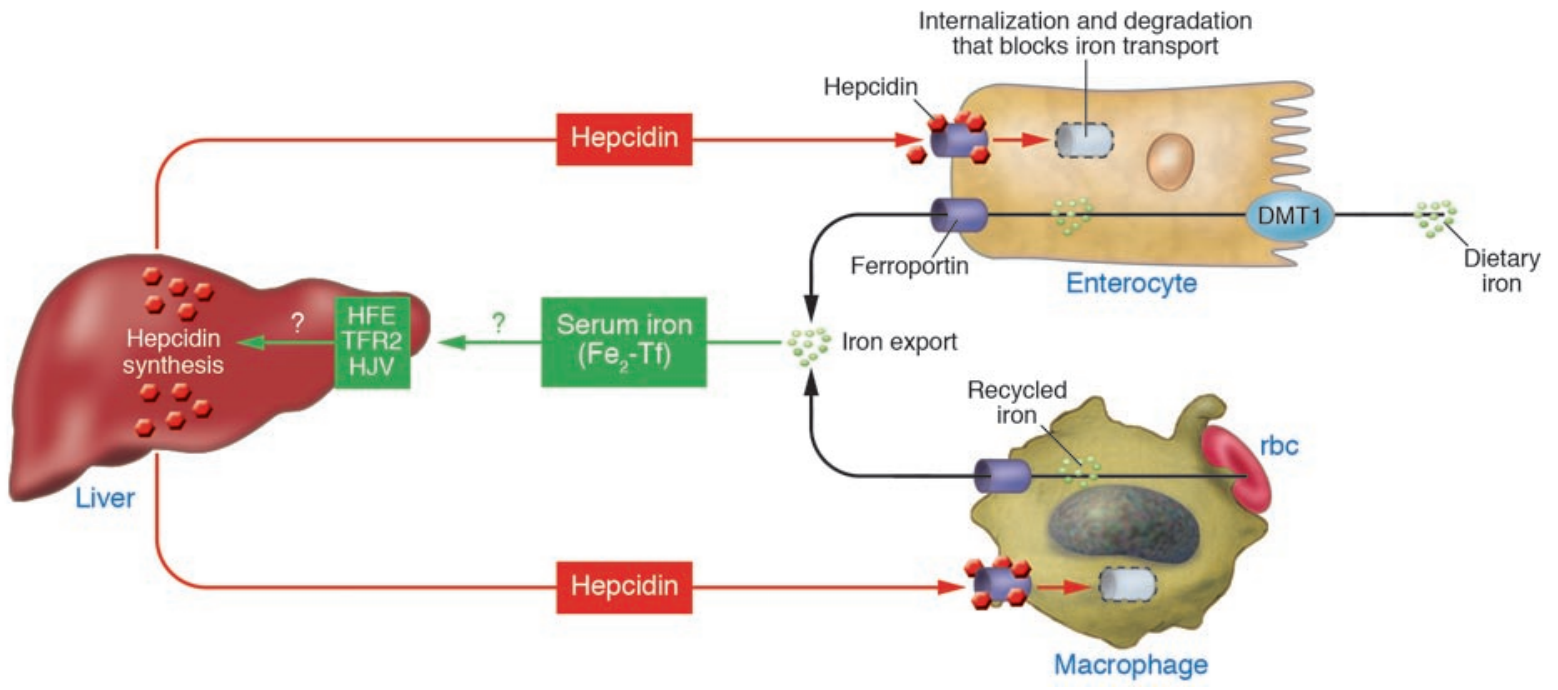

\section{Figure 1}

Regulation of systemic iron homeostasis. Increased diferric transferrin, $\mathrm{Fe}_{2}$ - $\mathrm{Tf}$, is detected by the liver via an as-yet unknown complex regulatory pathway involving HFE, TFR2, and HJV. Hepatocytes respond to this signal by inducing HAMP expression and hepcidin secretion. Circulating hepcidin acts in turn to diminish dietary iron absorption by the enterocytes and iron recycling by the macrophages through the internalization of ferroportin, which blocks iron export. As a consequence, serum iron decreases. As a feedback response, hepcidin synthesis is downregulated, which allows ferroportin molecules to be displayed on the surface of the target cells. In classical and juvenile hemochromatosis the mutations in HFE, TFR2, and HJV lead to abnormal hepcidin regulation, hypohepcidinemia, and ferroportin hyperactivity. The latter results in increased iron absorption and uncontrolled iron release from macrophages, 2 defects characteristic of hereditary hemochromatosis. DMT1, divalent metal transporter 1.

cellular uptake of transferrin-bound iron in most cells (reviewed in ref. 8). While the functional role of HFE remains a mystery [apart from regulating hepcidin antimicrobial peptide (HAMP) expression; see below], TFR2 was recently proposed to be involved in sensing serum diferric transferrin concentration at the level of the hepatocytes where its expression is directly regulated by holotransferrin $(9,10)$. Knockout mice lacking either Hfe (11-13) or Tfr2 (14) develop hepatic iron overload and increased transferrin saturation, as do human patients.

Juvenile hemochromatosis is a rare form of the disease characterized by the early and severe onset of symptoms, in particular cardiac and endocrine defects. Most juvenile hemochromatosis families have been reported to have mutations in the recently cloned $H J V$ gene (reviewed in ref. 8). $H J V$ is expressed in the muscle, liver, and heart, but the normal function of the protein is unknown (1). Several protein domains have been described, including a large repulsive guidance molecule (RGM) motif homologous to motifs of proteins involved in neuronal cell migration and a von Willebrand type D domain, but none of these domains have a known connection with iron metabolism (1). For a very small subset of patients, mutations have been identified in $H A M P$, which encodes hepcidin (reviewed in ref. 8). Hepcidin is a recently discovered secreted peptide synthesized mainly by the liver and excreted by the kidneys. It is a major regulator of iron metabolism and is positively regulated by iron and negatively regulated by iron deficiency, anemia, and hypoxia (reviewed in ref. 15). Hepcidin limits intestinal iron absorption as well as iron release by macrophages. Accordingly, hepcidin-deficient mice have been found to develop hemochromatosis with iron deposition in the liver and pancreas and, in contrast, decreased iron load in the macrophage-rich spleen (16). Conversely, transgenic mice overexpressing hepcidin present with severe iron-deficient anemia (17). The mechanism of hepcidin action has been recently determined $(18,19)$. To limit iron export, hepcidin binds to ferroportin, a transmembrane iron transporter necessary for iron transfer out of intestinal epithelial cells and macrophages (20), and induces its internalization and subsequent degradation (18). This action is expected to decrease dietary iron absorption in the enterocytes and iron recycling in the macrophages (Figure 1).

A breakthrough in the understanding of iron overload in hemochromatosis came first from the discovery that hepcidin production was deficient (as assessed by urinary hepcidin and/or liver mRNA levels) in mice and humans with classical hereditary hemochromatosis (21-27). Patients with juvenile hemochromatosis caused by mutations in $H J V$ were subsequently shown to be devoid of any measurable hepcidin in their urine (1). Therefore the concept arose that a regulatory iron-sensing pathway is centered around the hepatocytes, with HFE, TFR2, and HJV serving as regulators of hepcidin production by as-yet unknown direct or indirect mechanisms (Figure 1). Consequently, hereditary hemochromatosis severity could depend mainly on the level of residual hepcidin. Partial hepcidin deficiency would result in classical hereditary hemochromatosis, while almost complete deficiency would lead to juvenile hemochromatosis. In full agreement with this hypothesis, constitutive hepcidin expression has been shown to be able to prevent increased iron deposition in $\mathrm{Hfe}$ knockout mice (24).

The ferroportin disease is the third class of hereditary hemochromatosis. It is an autosomal-dominant disease caused by pathogenic mutations in the gene encoding the iron exporter ferroportin (reviewed in ref. 28). Since ferroportin is a down- 
stream target of hepcidin, hepcidin level is not expected to be decreased in this type of hemochromatosis (29).

\section{Juvenile hemochromatosis in $\mathrm{Hjv}^{-1-}$ mice results from impaired hepcidin synthesis and consequent overexpression of ferroportin}

In this issue of the JCI, Huang et al. (2) and Niederkofler et al. (3) report the establishment of murine models of HJV deficiency. Although the data presented do not provide any new information on the functional role of HJV, they effectively describe the increased iron deposition in the liver, heart, and pancreas and the decreased iron levels in tissue macrophages of the mutant mouse. The authors note that liver iron accumulation begins earlier and progresses more quickly in $\mathrm{Hjv}^{-/-}$mice than in $\mathrm{Hfe}^{-/-}$mice. The heterozygous $\mathrm{Hjv}^{+/-}$mice are indistinguishable from control mice (2). These results definitively confirm that lossof-function mutations in the Hjv gene lead to severe hemochromatosis and are consistent with the fact that parents of juvenile hemochromatosis patients are unaffected. Interestingly, both groups report that $H A M P$ mRNA is virtually undetectable in the livers of mutant mice, which suggests that HJV acts by depressing HAMP gene activity. It is likely that $\mathrm{HJV}$, like HFE, is needed as a sensor or mediator of activating signals of HAMP expression, including iron. In fact, the hepcidin gene's decreased sensitivity to iron has been reported in $\mathrm{Hfe}^{-/-}$mice (24). Niederkofler et al. show that the Hjv $v^{-/}$ mice fail to express $H A M P$ in response to dietary or injected iron, which demonstrates that the transmission of the iron signal in the liver is completely blocked in the absence of HJV. However, the authors report that $H \mathrm{Hv}^{-/-}$mice retain their ability to upregulate $H A M P$ expression in response to inflammation and emphasize that the 2 inducers of HAMP expression, iron and inflammation, act through different pathways, the latter one independent of HJV.

Having established the impairment of hepcidin synthesis in mutant mice, Huang et al. (2) further show that the ferroportin level is considerably enhanced in both intestinal epithelial cells and macrophages of $\mathrm{Hjv}^{-/-}$mice, most likely reflecting the profound hepcidin deficiency. Accordingly, ferroportin has been shown to be overexpressed in the intestine and macro- phages of hepcidin-deficient mice (30). It should be noted that in addition to hepcidin deficiency-induced ferroportin hyperactivity, Drakesmith et al. recently reported that ferroportin mutations resulting in insensitivity to hepcidin could also lead to ferroportin hyperactivity and subsequent hemochromatosis (31). Together, these results confirm and extend the key role of the hepcidin/ferroportin axis in regulating iron absorption by the intestine and iron efflux from macrophages.

While HJV mouse models of juvenile hemochromatosis are elegantly described in these important studies $(2,3)$, some key questions are still awaiting answers: (a) What is the functional role of HJV, in particular its role - if any - in the skeletal and cardiac muscles that express Hjv at a high level? Will older knockout animals develop specific muscular damage in addition to iron overload?; (b) Does hepcidin gene expression in organs other than the liver (e.g., in the heart, pancreas, and kidney) play any role? Does HJV regulate $H A M P$ expression in a similar manner in all tissues?; and (c) How do iron-sensing and inflammatory pathways converge on $H A M P$ expression, and at what level is the $H A M P$ /iron-activating signal disturbed in the absence of HJV? Could HAMP be activated in patients and mice suffering from this type of juvenile hemochromatosis by drugs that, for instance, mimic inflammatory cues but are devoid of their detrimental effects?

There is no doubt that the discoveries of the hormonal iron-regulating role of hepcidin $(16,17)$ followed by the elucidation of its mechanism of action - to regulate ferroportin degradation in the intestine, macrophages, and probably placenta (18) - herald exciting times in the field of iron metabolism and related disorders. The hepcidin/ferroportin axis occupies a central position in regulating iron homeostasis and explaining the physiopathology of iron-overload syndromes and likely - at the other end of the spectrum of iron disorders - anemia of inflammation (23, 32). Although considerable efforts are still required in order to solve remaining questions, the studies by Huang et al. and Niederkofler et al. have paved the way for rapid progress in the development of inducers or drugs capable of enhancing endogenous HAMP expression in spite of HFE or HJV deficiency. This should lead to breakthrough treatments for hereditary hemochromatosis, secondary hemosiderosis, and chronic inflammatory anemias (thanks to hepcidin antagonists or inhibitors of HAMP gene expression) - pathologies that affect millions of patients around the world.

Address correspondence to: Sophie Vaulont, Institut Cochin, INSERM 567, CNRS 8104, Université Paris 5, Paris 75014, France. Phone: 33-144-41-24-08; Fax: 33-144-41-24-21; E-mail:vaulont@cochin.inserm.fr.

1. Papanikolaou, G., et al. 2004. Mutations in HFE2 cause iron overload in chromosome 1q-linked juvenile hemochromatosis. Nat. Genet. 36:77-82.

2. Huang, F.W., Pinkus, J.L., Pinkus, G.S., Fleming, M.D., and Andrews, N.C. 2005. A mouse model of juvenile hemochromatosis. J. Clin. Invest. 115:2187-2191. doi:10.1172/JCI25049.

3. Niederkofler, V., Salie, R., and Arber, S. 2005. Hemojuvelin is essential for dietary iron sensing, and its mutation leads to severe iron overload. J. Clin. Invest. 115:2180-2186. doi:10.1172/JCI25683.

4. Andrews, N.C. 2005. Molecular control of iron metabolism. Best Pract. Res. Clin. Haematol. 18:159-169.

5. Pietrangelo, A. 2004. Hereditary hemochromatosis--a new look at an old disease. N. Engl. J. Med. 350:2383-2397.

6. Feder, J.N., et al. 1996. A novel MHC class I-like gene is mutated in patients with hereditary haemochromatosis. Nat. Genet. 13:399-408.

7. Camaschella, C., et al. 2000. The gene TFR2 is mutated in a new type of haemochromatosis mapping to 7q22. Nat. Genet. 25:14-15.

8. Roetto, A., and Camaschella, C. 2005. New insights into iron homeostasis through the study of nonHFE hereditary haemochromatosis. Best Pract. Res. Clin. Haematol. 18:235-250.

9. Johnson, M.B., and Enns, C.A. 2004. Diferric transferrin regulates transferrin receptor 2 protein stability. Blood. 104:4287-4293.

10. Robb, A., and Wessling-Resnick, M. 2004. Regulation of transferrin receptor 2 protein levels by transferrin. Blood. 104:4294-4299.

11. Zhou, X.Y., et al. 1998. HFE gene knockout produces mouse model of hereditary hemochromatosis. Proc. Natl. Acad. Sci. U. S. A. 95:2492-2497.

12. Levy, J.E., Montross, L.K., Cohen, D.E., Fleming, M.D., and Andrews, N.C. 1999. The C282Y mutation causing hereditary hemochromatosis does not produce a null allele. Blood. 94:9-11.

13. Bahram, S., et al. 1999. Experimental hemochromatosis due to MHC class I HFE deficiency: immune status and iron metabolism. Proc. Natl. Acad. Sci. U. S. A. 96:13312-13317.

14. Fleming, R.E., et al. 2002. Targeted mutagenesis of the murine transferrin receptor- 2 gene produces hemochromatosis. Proc. Natl. Acad. Sci. U. S. A. 99:10653-10658.

15. Ganz, T. 2005. Hepcidin-a regulator of intestinal iron absorption and iron recycling by macrophages. Best Pract. Res. Clin. Haematol. 18:171-182.

16. Nicolas, G., et al. 2001. Lack of hepcidin gene expression and severe tissue iron overload in upstream stimulatory factor 2 (USF2) knockout mice. Proc. Natl. Acad. Sci. U. S. A. 98:8780-8785.

17. Nicolas, G., et al. 2002. Severe iron deficiency anemia in transgenic mice expressing liver hepcidin. Proc. Natl. Acad. Sci. U. S. A. 99:4596-4601.

18. Nemeth, E., et al. 2004. Hepcidin regulates cellular iron efflux by binding to ferroportin and inducing its internalization. Science. 306:2090-2093.

19. Knutson, M.D., Oukka, M., Koss, L.M., Aydemir, F., and Wessling-Resnick, M. 2005. Iron release from macrophages after erythrophagocytosis is 


\section{commentaries}

up-regulated by ferroportin 1 overexpression and down-regulated by hepcidin. Proc. Natl. Acad. Sci. U. S. A. 102:1324-1328.

20. Donovan, A., et al. 2005. The iron exporter ferroportin (Slc40a1) is essential for iron homeostasis. Cell Metabolism. 1:191-200.

21. Ahmad, K.A., et al. 2002. Decreased liver hepcidin expression in the hfe knockout mouse. Blood Cells Mol. Dis. 29:361-366.

22. Bridle, K.R., et al. 2003. Disrupted hepcidin regulation in HFE-associated haemochromatosis and the liver as a regulator of body iron homeostasis. Lancet. 361:669-673.

23. Nemeth, E., et al. 2003. Hepcidin, a putative mediator of anemia of inflammation, is a type II acute- phase protein. Blood. 101:2461-2463.

24. Nicolas, G., et al. 2003. Constitutive hepcidin expression prevents iron overload in a mouse model of hemochromatosis. Nat. Genet. 34:97-101.

25. Muckenthaler, M., et al. 2003. Regulatory defects in liver and intestine implicate abnormal hepcidin and Cybrd 1 expression in mouse hemochromatosis. Nat. Genet. 34:102-107.

26. Kawabata, H., et al. 2005. Expression of hepcidin is down-regulated in TfR2 mutant mice manifesting a phenotype of hereditary hemochromatosis. Blood. 105:376-381.

27. Nemeth, E., Roetto, A., Garozzo, G., Ganz, T., and Camaschella, C. 2004. Hepcidin is decreased in TFR2-hemochromatosis. Blood. 105:4103-4105.
28. Pietrangelo, A. 2004. The ferroportin disease. Blood Cells Mol. Dis. 32:131-138.

29. Papanikolaou, G., et al. 2005. Hepcidin in iron overload disorders. Blood. 105:4103-4105.

30. Viatte, L., et al. 2005. Deregulation of proteins involved in iron metabolism in hepcidin-deficient mice. Blood. 105:4861-4864.

31. Drakesmith, H., et al. 2005. Resistance to hepcidin is conferred by hemochromatosis-associated mutations of ferroportin. Blood. doi:10.1182/blood2005-02-0561.

32. Nicolas, G., et al. 2002. The gene encoding the iron regulatory peptide hepcidin is regulated by anemia, hypoxia, and inflammation. J. Clin. Invest. 110:1037-1044. doi:10.1172/JCI200215686. 